Andrew M. Colombo-Dougovito*

\title{
The role of dynamic systems theory in motor development research: how does theory inform practice and what are the potential implications for autism spectrum disorder?
}

DOI 10.1515/ijdhd-2016-0015

Received February 24, 2016; accepted June 29, 2016; previously published online August 10, 2016

\begin{abstract}
Dynamic systems theory (DST) outlines three constraints (i.e. individual, task, and environment) that influence the emergence of behavior. These constraints interact with one another to self-organize and create a spontaneous behavior. For many researchers studying motor development, this spontaneous behavior refers to the production of motor movement. DST provides an explanation for the variability and spontaneous movement that occurs from individual to individual. While this theory is accepted as one of the major explanations of motor development, it is unknown how it is being utilized to inform the research on motor development or the development of interventions. In this review, the author found 18 instances in the literature where DST had been used to analyze, test, or manipulate motor patterns and movement. Overall, the studies report a positive effect from the manipulation of constraints with respect to a change in motor pattern. Only one study was found that sought to positively improve behavior through the directed use of constraints; the majority of studies sought to understand the influence constraints have on the production of movement.
\end{abstract}

Keywords: autism spectrum disorder; constraints; dynamic systems theory; motor development.

\section{Introduction}

The coordination of the human body to produce movement is a complex, systematic process [1]. This occurs largely unnoticed by the individual performing the

\footnotetext{
*Corresponding author: Andrew M. Colombo-Dougovito, Department of Kinesiology, University of Virginia, 210 Emmet St. S P.O. Box 407400, Charlottesville, VA 22901, USA,

E-mail: amcd@virginia.edu.http://orcid.org/0000-0002-5671-8826
}

movement and refers to the underlying processes of an individual's motor ability; often, the emergence, change, and growth of those abilities are considered motor development [2]. The idea of studying the dynamics of motor development in individuals is a research focus lacking great depth in understanding, relatively speaking in the context of research on human behavior. However, it has deep-seeded roots within larger fields of developmental research as far back as the late 1700s [3], including work from Darwin [4] and Shinn [5]. Early work in motor development was completed, primarily, by child psychologists in an attempt to comprehend the "nature versus nurture" phenomenon [6]. By the mid-20th century, motor development researchers seemed to have learned everything there was to know about motor behavior, and by the 1960s, researchers moved away from the biology of how motor movements were occurring toward more psychological aspects of cognition, language, and social development [7]. Nearly 30 years later, researchers reinvigorated motor development research, questioning the traditional views of how motor skills are thought to be attained and developed throughout the lifespan [8].

\section{A brief history of motor development}

Clark and Whitall [9] suggested four clear divisions in the focuses and theories driving thought in motor development. These were the Precursor Period (1789-1928), the Maturation Period (1928-1946), the Normative/Descriptive Period (1946-1970), and the Process-Oriented Period (1970-present). In the most recent developmental period of research, the Process-Oriented Period moved from predominantly focusing on what an individual can do to how an individual can do it. Early thinking in this period focused on information-processing theory, which, stemming from maturational theory, suggested the human brain functioned similarly to a computer in that movement processes are called up by the brain for the body to perform [10]. Once an individual learns the process of a 
movement, that information is stored to be recalled when needed. Realistically, this discrete, linear development of the brain does not fully cover the complexity that is human movement $[1,11]$.

Thelen and Ulrich [11] questioned the neuromaturational explanations of causation and cite the central issue with this prospective as limiting the scope of how movement is derived through behaviors arising from the interaction of many underlying subsystems and processes. While each discipline of thought has contributed multiple theories providing unique and crucial insight, often, these theories are focused on one singular aspect of development [12]. This growth of, typically, incompatible theories is as daunting to many development specialists as it is to practitioners attempting to use them, largely due to the little shared similarities [12]. An alternative theoretical explanation offers a multi-faceted approach to motor development and is referred to by a variety of terms, including coordinative structure theory, dynamical perspective, dynamical systems, dynamical pattern theory, ecological approach, or constrain-based/ led approach [13], but is more predominately known as dynamic systems theory [14].

\section{Dynamic systems theory}

The concept of dynamic systems is popular across a multitude of fields, including, but not limited to, mathematics, physics, astronomy, chemistry, meteorology, and the biological, cognitive, neurological, and social sciences [11], and most recently, in the fields of physical and occupational therapy. Dynamic systems theory (DST) $[14,15]$ suggests that a behavior occurs as the confluence resulting from interactions of the characteristics of the individual, environment, and task (see Figure 1). These are commonly referred to as constraints. Newell $[14,15]$ outlined that it was constraints that allowed for the coordination of a behavior within an individual and allowed that behavior to emerge. Often, when spoken of in the motor development

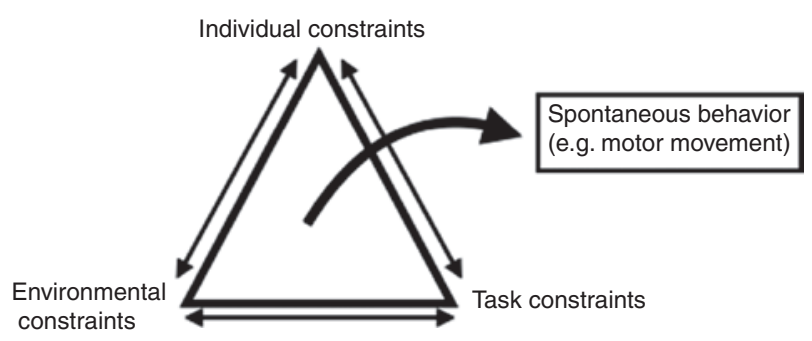

Figure 1: Newell's model of dynamic systems theory. domain, this behavior is referred to as movement; however, the interaction between the three constraints could result in any form of behavior, not just movement.

It is from this framework that many developmental specialists studying motor behavior (see the collective published works of Jane Clark and Ester Thelen) in individuals have explained the complex coordination of all the influences on movement. The term individual is more common in recent literature over Newell's term organismic [14], mostly due to relating the theory to human movement as opposed to the general, bio-mechanical definition given by Newell. Individual constraints are often considered to be the structural (e.g. weight, height, etc.) and functional (e.g. motivation, attention, etc.) characteristics that a person possesses unique to him or herself [16]. Therefore, environmental constraints refer to everything that exists outside of the individual; for example, the temperature, time of day, space (e.g. inside or outside), or the surface of the floor/ground could all be considered environmental constraints [17]. Hutzler [18] takes environmental constraints a step further, including social (e.g. peer, parent, and professional attitudes and support) and physical barriers (i.e. accessibility). Lastly, task constraints encompass everything involved in the action itself. These constraints could include the directions of the task (e.g. keeping personal space), the movement goals (e.g. doing something very quickly or slowly), or the equipment being used [19]. Often seen as a negative term synonymous with restraints, constraints under DST are viewed neither as negative or positive but a neutral term referring to the influence on behavior (in this case, motor movement) to encourage the production, and over time, development of that behavior [19]. This seemingly has a greater appeal to understanding the complexities of human movement, as throughout the lifespan, new behaviors emerge, evolve, and, on occasion, dissolve [13], but most importantly, have difficulty manifesting themselves the exact same way twice [20].

Furthermore, DST [14] changes the focus of movement from being a program that is run or performed to an emergent behavior based on the coordination of the various degrees of freedom. By accounting for influences from the constraints within the individual, the environment, and the task itself, the body is able to coordinate movement. If the brain was left to control each of the degrees of freedom, humans would be limited to one singular movement or task at a time. Take walking from a concrete surface onto an icy surface, for example. If the brain was the sole provider of information for movement, by the time the brain recognizes what is occurring, the individual would have already fallen to the ground. In contrast, DST suggests that most action occurs within the 
central nervous system, and based on the influence from constraints, explores all potential possibilities, settling on the most effective (i.e. attractor state) based on those constraints [21]. Thus, when the individual steps onto an icy patch, this new constraint alters the other constraints and the individual shifts his/her motion to remain at equilibrium (e.g. standing upright and moving forward).

DST builds upon research from Bernstein [22], which rejects motor movement as being a simple progression of one skill to the next. Bernstein defined movement in terms of coordination, the cooperative interaction of multiple body parts, and processes to produce a unified outcome [23]. Further, Bernstein ([22], as cited in Thelen [7]) argued that researchers must reject the notion of a one-to-one relationship of "neural codes, the precise firing of the motor neurons, and the actual movement pattern". In order for movement to occur and for the body to coordinate the potentially infinite combination of muscles, nerves, cells, as well as body segments and joints, there must be external forces outside of the brain's control influencing behavior and restricting the degrees of freedom [22]. According to Newell [14], constraints are how certain degrees of freedom (e.g. the possibilities of movement in any given system) are limited. Constraints act as vital limiters to movement and influence efficient, effective patterns of movement.

In DST, the spontaneous pattern formation that emerges from the interaction of constraints is considered to be self-organizing $[1,7,24]$. Self-organization is the body's ability to find a stable pattern of movement based on the influence of constraints [20]; these stable states are often referred to as attractor states $[7,11]$. An attractor state refers to the preferable pattern based on a set of constraints [7,25], but it is not the obligatory pattern [1]. As the influence of constraints is a constantly evolving process, constraints evolve, gaining and losing importance in their ability to influence movement. Behavior (i.e. movement) is dynamic and adaptive; there are multiple, redundant pathways to reaching the same goal [26] and learning takes place through patterns emerging according to the affordances and constraints [18, 21]. Therefore, the changes in motor ability, within a dynamic systems model, are considered to be nonlinear with step changes being the norm [12], meaning that as one constraint changes, that constraint, in turn, affects the growth and influence of the other constraints, producing a new movement pattern [19, 27]. Additionally, not one subsystem of constraints holds privilege, nor contains all the elements for the skill [1]. Therefore, by changing one or more of the parameters of movement for an individual, DST predicts that subsequent adaptation of the remaining constraints will be adjusted, resulting in a change in behavior $[13,25]$. This provides a potentially more in-depth mode of modifying movement, than previous theories. Researchers and practitioners can evaluate the whole situation to better understand what is acting as a limiter within the movement and analyze what needs to be addressed through intervention.

\section{Implications for individuals with autism spectrum disorder}

The individual influence from each constraint within DST offers insight into the motor development of every individual, but provides a particularly useful insight into the movement patterns of individuals with disabilities. Instead of looking at disability as a deficit that needs to be overcome, DST suggests that disability is simply acting as an individual constraint which is influencing that person's movement [19]. By adjusting the viewpoint of disability, building motor skills becomes less about overcoming barriers and more on adapting constraints to encourage more efficient movement patterns. In understanding that one constraint influences another to allow for movement to emerge, by adjusting or modifying tasks or the environment, a practitioner or researcher can create a situation for a more appropriate motor movement to occur [28].

This idea may have great benefit in the area of autism spectrum disorders (ASD). Children with ASD have been shown to have motor development patterns that differ from typically developing peers $[29,30]$, as well as developmentally matched peers [31]. Further, delays in motor ability appear to occur early in development $[32,33]$ and become greater as they age compared to peers [34]. As motor development research is a relatively new focus of research in the field of ASD, it is generally lacking depth [35]. Much of the research has focused on understanding the general delay displayed by children with ASD (see Fournier, Hass, Naik, Lodha, and Cauraugh [36] for an overview); however, impairments do not appear to be totally universal [37].

An effective method for improving the motor abilities of children with ASD is lacking. As much of the research on ASD has focused on improving social communicative deficits and repetitive behaviors [38], the hallmark of ASD diagnosis [39], there has been little done beyond making modifications based on previous evidence-based practices [40] or modifications to better include children in activity [41], as well as to improve performance on motor assessments [42, 43]. While each of these is necessary, a dedicated intervention built on a sound theory is needed 
to assist in building necessary skills so that children can be effectively included with peers, which will ideally increase the likelihood of sustained physical activity over time [44] and social opportunity [45]. Furthermore, interventions for young children with ASD may have a potential to help with language development [46].

DST may provide the guiding insight into how this intervention should appear and how constraints can be modified to make meaningful change in the motor ability of individuals. Although the efficacy of DST has not been formally tested, there are examples of how utilizing constraints has shaped behavior [20], even if those doing the work did not fully realize their inclusion of DST. By modifying constraints of a task or within the environment, an intervention based in DST has the ability to move past what typically limits instruction for children with ASD. Newell [14] suggested that 'extreme manipulation' is needed to further test the notions of the self-organization of coordination. Further, Newell and Jordan [15] recommended further research was necessary to understand how the theory worked and could be implemented.

\section{Purpose}

As there is little known about how motor intervention research has been impacted by DST and a lack of motor interventions for ASD, a comprehensive literature review was conducted. The purpose of this review was to analyze how, if at all, DST is being used in motor intervention. Moreover, how is DST informing the practice of building interventions focused on the building of motor skills? Additionally, how has DST been altered to address the motor development needs of individuals with disabilities, specifically ASD? This review focused on published research literature on motor intervention and DST by describing study characteristics and any major findings.

\section{Methods}

The following databases were searched for relevant studies: Academic Search Complete, Education Full Text, Education Research Complete, ERIC, Google Scholar, Physical Education Info, PsycINFO, ProQuest, PubMed, PubMed Central, SPORTDiscus, and ScienceDirect. Article references were searched for additional eligible studies. Studies were identified by searching each of the identified electronic databases and scanning reference lists of identified articles. The search included three lines of search words, including: (a) dynamic systems theory, ecological approach, constraint-led approach; (b) motor development, motor behavior, motor learning, motor skills, gross motor activities, fundamental motor skills; and (c) youth, children, adolescents. Initial keyword searches analyzing the whole text for terms resulted in nearly 20,000 results; however, in review of these early searches, the author realized that the databases were returning articles simply mentioning 'dynamic' or 'approach', not the theory itself. As this review is focused on DST, it is likely that these terms would be included in the abstract, title, or keywords. So the first search term was limited to title, keyword, and abstract searches, while the remaining lines were left open to full text searches. This resulted in output that was much more focused to the purpose of this review. In order to account for any articles that were missed during database searches, search results were compared against articles citing Newell's 1986 article.

\section{Inclusion criteria}

Studies were included in this review if published in the English language in a peer review academic journal between 1986 and August 2015, in which motor tasks were the primary focus of the study and DST was referenced. Exclusion criteria for this study were publication in non-English, prior to 1985, in which motor tasks was not the primary focus and DST was not referenced. Additional exclusion criteria included unpublished theses or dissertations, and conference proceedings. Studies were limited to those post 1986, since this was the year in which DST was first introduced by Newell.

\section{Overview of articles included}

The full search process produced a total of 368 articles. A further search of articles citing Newell's article produced an additional 1246 articles. Of the 1561 articles found, 1483 studies were excluded for failure to meet the inclusion criteria; common reasons for excluding studies included article duplication, lack of dynamic systems references or theory, motor tasks not the outcome focus, domain outside of kinesiology (e.g. robotics or speech therapy), review or metaanalysis, and either practical or theoretical application of dynamic systems. After the initial search was completed, the author searched, full text, the remaining 78 qualifying articles for studies focusing of DST's application to motor skills or motor development. Studies were included that had a defined motor intervention searching to build gross motor skills that was definitively influenced by DST. A total of 18 articles were identified that met all criteria and were included for analysis. See Figure 2 for procedure by which studies were selected. Further, Table 1 contains the essential characteristics extracted from each article; Table 2 outlines the main findings of each study.

\section{Results}

\section{Study characteristics}

\section{Participants}

Of the 18 studies, 5 (28\%) focused on infants, 7 (39\%) used children, $5(28 \%)$ used young adults, and $1(5 \%)$ 


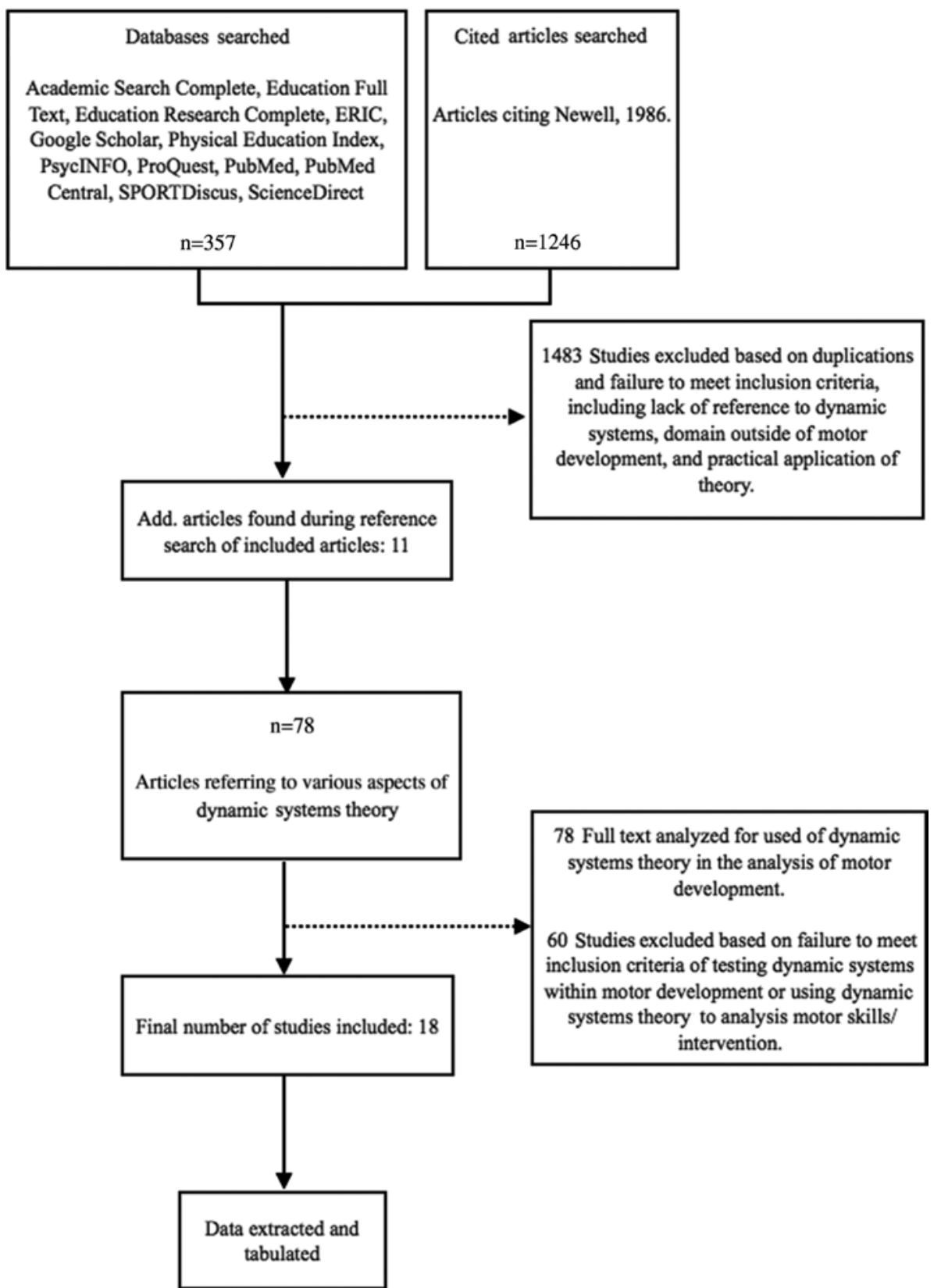

Figure 2: Flow of literature analysis.

used a mixed sample of both adults and children. Of the studies including children, ages ranged between 5 and 14, with the majority of children between 9 and 10 years of age. The studies with young adults focused on participants between the ages of 17 and 26. The mixed group study [52] utilized two separate groups, a young adult (ages between 19 and 25) and children (ages between 9 and 10); groups were analyzed separately, but visually compared for similarities in performance across age groups.

\section{Characteristics}

The majority of studies, 56\% $(n=10)$, included children or young adults with no disabilities or health concerns. Twenty-eight percent $(n=5)$ utilized either all participants with disabilities $(n=3)$ or participants with and without disabilities for comparison $(\mathrm{k}=2)$. Two studies in which all of the participants had a disability $[59,60]$ utilized infants with Down syndrome; the other [62] used children with congenital spastic hemiparesis, but no other comorbidities. 


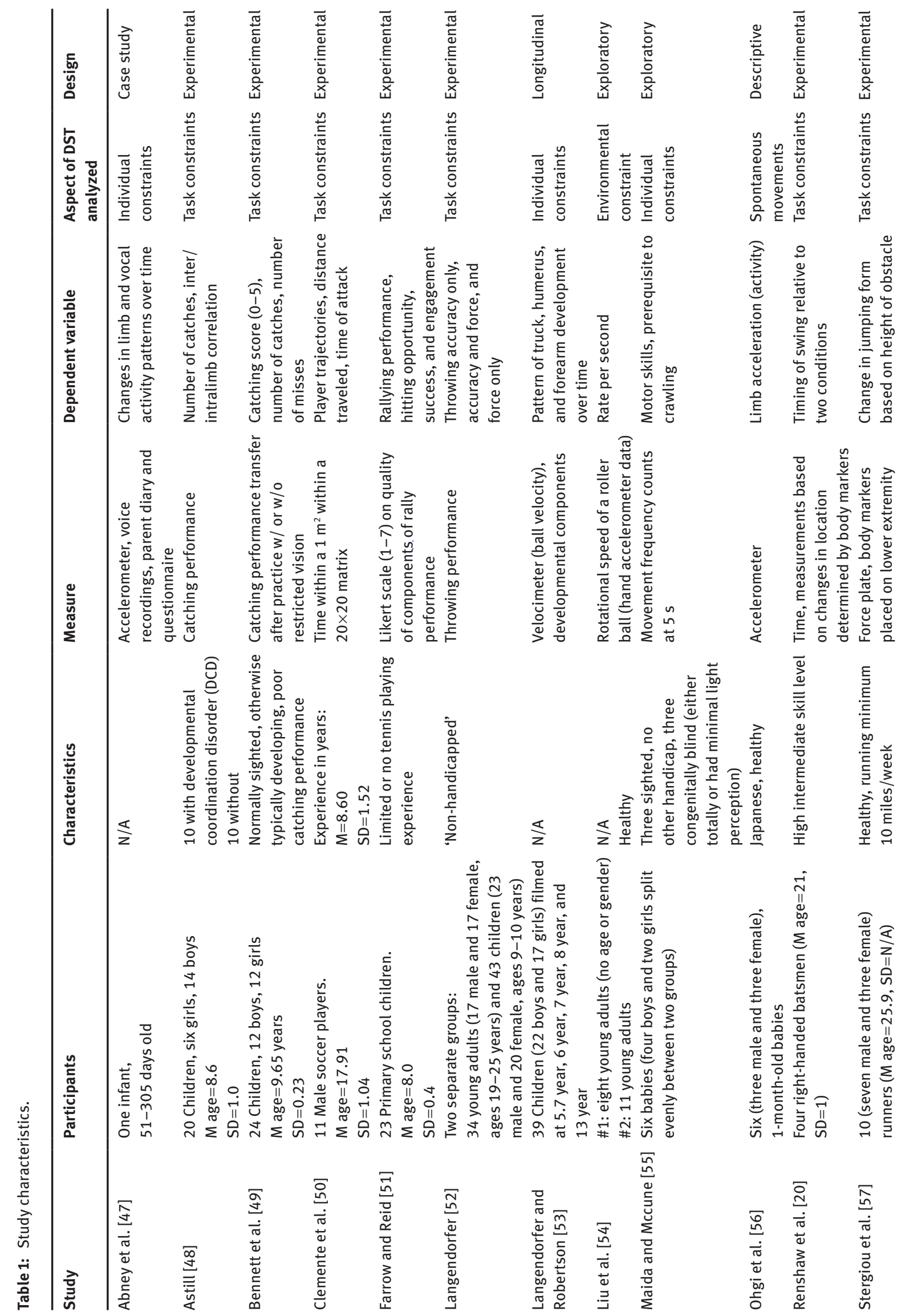


The two studies comparing participants with and without disabilities $[48,55]$ included children with and without developmental coordination disorder and visual impairments (defined as either totally blind or minimal light perception), respectively. No studies included individuals with ASD. Three (17\%) studies did not include enough information to ascertain the characteristics of the participants. In one of those studies [47], the participant was a 51-day-old infant at the commencement of the study; therefore, any physical disabilities would have been known. In another study [53], the data used was from an earlier collection in a previous study and aggregated to analyze longitudinally. It is likely that participant details were included in the original study; however, this was not referenced in this article. In the last study [58], children were included as intact classes; it is very likely that there were participants who have a disability, however, this information was not provided.

\section{Design}

A variety of research designs were used in this analysis: twelve $(67 \%)$ were experimental, two $(11 \%)$ were exploratory, two $(11 \%)$ were longitudinal, one $(5 \%)$ was descriptive, and one (5\%) was a case study. Of the studies that were experimental, all studies were focused on understanding the effect of a condition on an outcome variable. For example, in one study [21], researchers wanted to understand the effect of pitching condition (either machine or person) on the timing of each phase of a batter's swing.

\section{Aspect of DST analyzed}

Across the included studies, all of the aspects of DST were covered; however, as others have suggested [15], task constraints were the most commonly analyzed in the included studies. Of the 18 studies, 11 (61\%) analyzed task constraints or used task constraints to manipulate behavior. Four of the studies (22\%) focused on individual constraints to understand the development of behaviors or how behaviors emerged due to changes in individual constraints. Of the remaining, two studies focused on environmental constraints and one study analyzed the overall spontaneous movement occurring in infants, not attributing it to any one constraint in analysis.

\section{Dependent variable}

Each of the studies included focused on a change of behavior, whether it was a change in limb patterns $(n=4)$, 
Table 2: Major findings.

\begin{tabular}{ll}
\hline Study & Major findings \\
\hline Abney et al. [47] & $\begin{array}{l}\text { Changes in vocalization were preceded by a period of higher variability. Limb activity increases; leg activity } \\
\text { becomes more stable and repetitive with age, while arm pattern demonstrated an inverse relationship }\end{array}$ \\
Astill [48] & $\begin{array}{l}\text { Changes in the task (i.e. ball thrown at midline, right, or left) changed the outcome for children with DCD. } \\
\text { Children were much more likely to be successful, not only in catching the ball, but coordinating his/her } \\
\text { limbs to catch, when the ball was thrown toward the midline or right side }\end{array}$ \\
Bennett et al. [49] & $\begin{array}{l}\text { Participants demonstrated an increase in the number of catches and decrease in the number of misses, } \\
\text { regardless of group. Practice performance varied the greatest under the varied visual manipulations }\end{array}$ \\
Clemente et al. [50] & $\begin{array}{l}\text { The type of tasks (i.e. risk, neutral, or conservative) influenced the movement and time taken for the } \\
\text { attacker. Attackers in a risk situation (e.g. team losing with time running out) drove the ball toward the goal } \\
\text { and took a shot much quicker than attackers in other situations }\end{array}$ \\
Farrow and Reid [51] & $\begin{array}{l}\text { A scaled court provided an increased performance, regardless of scaled ball size. Participants using the } \\
\text { standardized adult conditions demonstrated decreased hitting opportunities, as well as lower success and } \\
\text { engagement }\end{array}$
\end{tabular}

Langendorfer [52]

Some throwers demonstrate different patterns to achieve different throwing goals. Male patterns showed greater change under different conditions. Age played very little role in the change of patterns

Langendorfer and

Robertson [53]

Participants demonstrated commonalities in development in both order of developmental levels and common pathways of development; however, there were individual differences. Differences were attributed to the interaction of constraints for those particular emergences of patterns

Liu et al. [54]

Three levels of learners emerged; the most successful level of learning resulted in an S-shaped behavioral outcome, showing a sudden jump in performance. Another group demonstrated a much more level learning pattern and one group showed no performance increase. Different patterns of change are depended on the task dynamics and the learner

Maida and Mccune [55]

Individual differences in the pattern of development in many of the categories, however, demonstrated an underlying sequence with variations of length prior to advancement

Ohgi et al. [56]

Motor development in infants occurs through processes of self-orientation, suggesting a nonlinear system in contrast with the traditional view of infant behavior as simple reflexes. Infants' spontaneous movements are influenced by constraints capable of voluntary skilled movement

Renshaw et al. [20]

Swing patterns changed significantly between different environmental constraints

Stergiou et al. [57]

The height of an obstacle caused a change in patterns of behavior; however, the variability of the system remained constant, meaning that although the pattern changed, the internal factors adjusted to maintain as little deviation from a typical pattern

Sweeting and Rink [58]

Environmental instructions did improve performance, suggesting that the use of environmental tasks to elicit performance is a viable instructional approach, but should not be used exclusively or indiscriminately

Ulrich et al. [59]

Step patterns varied depending on the task constraint provided. Provides evidence of how future interventions could influence stepping behavior through manipulating the task

Ulrich et al. [60]

Infants with DS were able to perform alternated walking steps when supported, long before walking voluntarily. Lower body fat, scaled-up strength, and ability to move forward act as control parameters alternative walking

Vernadakis et al. [61]

Task modification provides a useful tool to build object control skills. Exergaming provides a more enjoyable method of improving skills, while resulting in similar gains to a typical face-to-face administration

Volman et al. [62]

Applying a functional task context elicited positive changes in the control of reaching movement of the affected arm of children with spastic hemiparesis. Treatment of children with spastic hemiparesis should focus on practicing functionally relevant skills (actions) instead of non-functional movements

Wu et al. [63]

Task conditions (e.g. size and location) have an effect on movement time, peak velocity, and the percentage of time-to-peak velocity. The accuracy of an individual may also be affected by the individual's constraints, not only by the object's size or location

walking pattern $(n=3)$, aspects of throwing $(n=2)$, catching performance $(n=1)$, swing pattern $(n=1)$, prerequisite motor skills to crawling $(n=1)$, general fundamental motor skills $(\mathrm{n}=1)$, jumping distance $(\mathrm{n}=1)$, tennis rallying $(n=1)$, offensive soccer strategies $(n=1)$, or walking gait $(n=2)$. Only one study [61] utilized a standard assessment 
battery; the remaining studies used either an accelerometer (22\%), a count of frequency by coded behaviors (22\%), timing (5\%), a force plate (5\%), walking pattern analysis $(11 \%)$, or a product-oriented assessment (e.g. how many catches; $28 \%$ ). Four of the studies, in addition to other outcome measures, utilized body markers to analyze how the joints and limbs changed in relation to one another $[20,57,62,63]$.

\section{Major findings}

Each of the studies varied in their use of DST to explain the results or the interpretation of them. Overall, however, each of the studies in their own interpretation suggests a very strong influence of constraints on the motor movement of each of the included participants (See Table 2). Within infants, motor behaviors appear in a nonlinear fashion [56] with spontaneous movement becoming more stable and repetitive in the lower extremities, but with increasing variability within the upper limbs [47]. These findings suggest development is less reflexive during the early years and is driven by outside forces. The only anomaly within the infant studies was Maida and Mccune's [55] study on patterns of movement in infants. However, when analyzing this study, it became clear that they used DST in the design of the study, but analyzed the data with a maturational lens looking to confirm the presence of stages of development.

Several studies $[20,52,59,60]$ utilized dynamic systems to manipulate behavior in order to understand the influence of constraints. In each of the studies, behavior (i.e. motor performance or motor movement) spontaneously changed to accommodate the added constraint. For example, Ulrich and colleagues [59] tested the walking patterns of infants with Down syndrome when walking on different surfaces. These included walking on Velcro with socks and bare-foot on a bumpy surface. Each of the surfaces influenced a different movement pattern within the infants, suggesting that the motor behavior can be influenced to improve outcomes of an intervention. Further, Renshaw and colleagues [20] analyzed the swing pattern of young adult cricket players. In this study, the swing pattern and timing was analyzed when participants were swinging at a ball bowled from a machine or a person. Findings suggest that the timing was faster when a machine was pitching. This study demonstrates the spontaneous influence an environmental cue can have on a behavior, even when the overall situation is similar.

Only one study focused on an intervention [61]. In this recent study, the authors compared two motor interventions, one face-to-face and one exergaming (i.e. exercising to a video game), to a control group on the improvement of fundamental motor skills. The underlying premise of the intervention was developed around modifying the tasks of the skills to improve performance. By manipulating the task constraints for the individual, the authors demonstrated a significant improvement over the control group. There was not a significant difference in performance between the face-to-face and exergaming interventions other than enjoyment; participants in the exergaming group reported a higher enjoyment. Table 2 provides the major findings for each group.

Of the studies that included individuals with disabilities, the outcomes when utilizing constraints were positive. Ulrich and colleagues $[59,60]$ demonstrated a positive influence in the walking patterns when controlling for individual constraints and with imposed task constraints. Astill [48], with children with developmental coordination disorder (DCD), demonstrated the influence of certain task constraints for children with coordination issues. By controlling for where and how a ball is delivered, researchers saw an influence in the overall performance; suggesting that to improve success and performance, instructors should take task constraints into consideration. No studies were found that included children with ASD.

\section{Discussion}

The focus of this inquiry was to understand how DST [14] has been utilized to understand motor development or build motor skills through the analysis of the literature by describing common study characteristics and detailing major findings. As a result, this analysis summary provides an empirical insight into how DST has been used in research of motor development. First, research has demonstrated a great variance of the interpretation and inclusion of dynamic systems within the study purpose, design, and interpretations. Second, research with dynamic systems has focused very heavily on the very earlier years (infancy) of development often to simply give an explanation of patterns of movement. Third, with the exception of one study [61], there is a lack of evidence that this theory is being used in motor interventions. Lastly, with regard to the relevance to individuals with ASD, no past studies have included this population and only a few studies have included individuals with disabilities.

Beyond the variance of sample size and purpose, the included studies suggest the foundations of DST 
are present within the production of movement and give evidence for its use in improving motor skills and development. Ulrich and colleagues [59], in manipulating the surfaces of a treadmill, were able to manipulate the walking patterns of infants with Down syndrome. Further, the authors suggest that the results give evidence to the use of task manipulation to improve motor patterns. This evidence is further strengthened by Vernadikis and colleagues [61] in the intervention study utilizing task manipulation to improve motor skills. While the authors' ultimate goal was to understand the effects of exergaming in relation to a standard face-toface intervention, this study provides strong evidence for utilizing task manipulation to influence and improve the motor skills of children. Whereas evidence is limited in the overall literature, the studies included provide positive support for the use of task manipulation in motor intervention. The studies included in this review further support the belief that task manipulations are the most common constraint to enact change [15], as over 50\% of the studies included in this review assessed or used task constraints. Most commonly, studies utilized manipulations of equipment as a modification of a task [51, 52, 57, 59]; however, changes in the task instruction could also provide useful [50].

Individual constraints ( 4 of 18 studies) were included in looking to understand how movement is produced and, often, the development of early motor patterns in infants. As this is a difficult area to 'manipulate' within individuals, it is not surprising to see the lack of manipulation in this area. Ulrich and colleagues [60] manipulated the individual constraints of infants with Down syndrome by holding infants up while they 'walked' on a treadmill. In this study, infants when held demonstrated an effective, alternating walking pattern long before they demonstrated independent walking, demonstrating that a combination of individual constraints, like strength, can act as a rate limiter [7] preventing independent walking from occurring. Infants in this study were not able to produce a walking pattern until their leg strength, and likely balance, was scaled up to allow for independent walking. However, when the individual constraint of balance was manipulated, the walking pattern emerged in the infants. Manipulation of individual constraints can be fairly straight forward in infants, but to scale this for older population is slightly more difficult. A task analysis may provide insight into this issue [64-66]. A task analysis develops a breakdown of the skills in question from simple to complex, including everything needed to perform the skill. This may reveal that an individual is limited by strength when performing a basketball shot. A researcher or practitioner may then focus on scaling up the individual's strength to allow for a more mature pattern to emerge.

Lastly, environmental constraints comprised the fewest studies included in this review. This result is surprising, given the popularity of structured teaching and influence of the environment demonstrated in psychology. However, the environment is typically considered in addressing behaviors such as on-task/off-task or time-ontask. It could be that the focus is so often on the task or individual that the environment is assumed to be of little effect or 'controlled'. However, in Newell's model [14], each area of constraint plays an equally vital role in the emergence of behavior. Any change in the environment could result in a varied motor pattern. This area is in need of further review to understand how an individual's pattern of movement is changed based on the environment. Sweeting and Rink [58] demonstrated an improvement in jumping performance by utilizing an environmental constraint teaching model; however, the authors suggest it be used in conjuncture with other teaching strategies, not as a standalone method.

In this review, about a third of the research studies (5 of 18) were done with infants as participants. As the origins of motor development begin in infancy, it is logical that a considerable amount of research would focus on these key developmental years. Additionally, infant development had been thought to be predominately reflex driven and predominately thought of in stages, mostly due to much of the earlier work of maturational researchers $[67,68]$. However, few studies have gone beyond this age. From maturational research, there is an understanding that individuals develop throughout the lifespan and within each stage there are important skills to learn and develop [3, 69]. However, it is clear that many skills thought to occur due to maturation do not simply appear and need instruction [70]. Further, the variability between when individuals develop and how proficient they become at a skill does not fit in a maturational model. As several of the studies in this review have demonstrated, constraints retain influence as individuals age [52] and can have different effects on the emergence of movement patterns during the development process [53].

A finding that stands out in this review is the lack of intervention research done using DST as a framework, as there have been countless suggestive articles as to the relevancy of this theory to development and the need for research [71, 72]. One study [61] included in this review focused on the improvement of motor skills, not simply understanding the constraints' influence of motor performance. Several studies $[20,48,52-54,58,59]$ focused on the influence of the constraint which resulted in an altered 
performance. In this author's view, these are not dedicated interventions, as the researchers of those studies were not focused on designing improvement of skills, but on the influence of constraints; improvement happened as a result of the manipulation of the constraints, not due to any predetermined influence. The intervention designed by Vernadakis and colleagues [61] sought to improve object control (e.g. kicking, throwing, etc.) skills through using DST as a framework. This study provides an important example for the benefit in utilizing dynamic systems within an intervention.

Vernadakis et al. [61], to study the effects of an exergaming routine against a traditional activity (TA) group, developed two 8-week interventions. Both groups received the intervention twice per week for $30 \mathrm{~min}$ per session. No rationale was given for the length or timeframe; however, when looking at the intervention, it follows a typical physical education unit based on time per week and length. Within each intervention, critical elements of correct movement were imbedded into each lesson and a task analysis was done to inform the development of skills from simple to complex. Four lessons were developed for each intervention prior to beginning, but the rest were left open to allow for the flexibility to adjust to participant needs as skills emerged. In both interventions, the tasks were manipulated to encourage proper patterns of movement; within the TA group, equipment was also modified to further encourage new, appropriate patterns of movement. Ultimately, Vernadakis et al.'s goal was to test the potential validity of an exergaming motor intervention to a TA intervention to support the use of such an intervention. Unintentionally, or perhaps there was intention, the researchers provided strong evidence for the use of DST in the creation of intervention, as both interventions (i.e. exergaming and TA) demonstrated a significant improvement from a pre- to a post-test, when compared to a control. Further, pre-tests resulted in a non-significant difference between groups, but each group did show a delay in motor skills. This demonstrated that through DST, individuals with a motor delay can show a significant improvement over a short amount of time.

Vernadakis and colleagues [61] further demonstrate positive support for the use of dynamic systems in a motor intervention to increase motor skills; however, the authors also detail some of the potential issues in recreating this result. The researchers utilized a skilled, trained motor skills instructor to deliver instruction, as well as a task analysis to break the skill into a logical progression. Further, instruction was developed as the participants progressed, which is an important piece of this intervention. The instructors utilizing this intervention need to have the ability to visually assess and make the judgment of when to adjust the skill to fit the needs of the participant. As the types of modifications were not included, it is difficult to understand the detailed adjustments the instructor, or the exergame for that matter, made. Future interventions will need to pay special attention to this piece of the intervention because, as DST describes, the modification of the constraint must be strong enough to perturb the attractor well for that skill $[7,13]$. If the modification to the constraints is not powerful enough, individuals will simple return to the previous state of behavior.

With that being said, the study by Vernadakis et al. [61] is an encouraging outcome that is overdue and necessary to fully understand DST, as well as its effects on motor behavior [15]. This study provides an important step toward building more effective motor intervention for individuals with motor delays. As DST states that the influence of constraints allows for behavior to emerge from the central nervous system $[7,14,73]$ without necessary input from the brain, this type of intervention could potentially be very beneficial for individuals with cognitive or pervasive developmental disorders, such as individuals with ASD. Children with ASD have demonstrated that a delay is occurring in their motor behavior and their movement patterns are different compared to peers [29, 30, 36]. DST may provide the framework for the manipulation of constraints in order to move past deficits in communication and social behavior, as the constraints influence behavior beyond the control of the brain and beyond the influence of instruction [74]. As Ulrich, et al. [59] demonstrated with infants with Down syndrome walking with different constraints on a treadmill, the type of constraint can spontaneously influence the change in motor pattern. As long as the constraint has enough influence and the correct influence, interventions have the possibility to influence a positive change in behavior.

While research was not found utilizing DST for populations with ASD, the included studies provide a solid foundation for its use in the future. DST shifts the concept of disability from barriers that need to be overcome to individual constraints that influence behavior. This not only allows for the understanding of the limitations individuals face when trying to produce movement similar to someone without that disability, but it also recognizes that the individual's disability may also positively influence a person's movement in a way that someone without the disability could not do. Of the studies that included populations with disabilities, each provides positive support that, through the concepts of DST, individual movements can be influenced into a more mature movement pattern. Lastly, although a minor finding in Vernadakis et al.'s [61] 
study, an intervention built upon DST can provide positive support for individuals demonstrating a motor delay. As the evidence suggests that most individuals with ASD have a delay [36], providing an intervention by modifying constraints can have a potentially significant impact.

\section{Limitations}

As the field of motor development and motor behavior has similar origins and similar theories, this study acknowledges the potential limitation in limiting its search to only DST to understand how the theory of constraints is driving motor development. Gibson's Ecological Model [75] is common among motor learning research and provides strong evidence toward the use of analyzing the environment and the perception of the individual with regard to motor skill. Further, Schmit's Schema Theory [76] is also widely used and accepted as a model of skill development. However, these theories are interested in discrete task learning, not on lifespan development as a whole. DST describes how the influence of constraints allows for a motor behavior to emerge on a discrete skill basis; however, it also describes development as a whole being nonlinear and occurring longitudinally. Therefore, this review focused on DST exclusively.

Further, the author recognizes that this list may not be encompassing of all research utilizing constraints to development motor skills, as to a point where much of the research is likely to include some form of task, environment, or individual manipulation or modification. However, they may not fully understand where the basis of those modifications is coming from or base them in DST. Wicke and Jensen [27] is an example of a study excluded from the review for lack of inclusion of Newell's DST; in this article, the authors describe dynamic systems from the viewpoints of several referenced articles pertaining to a dynamic systems approach, but because these are secondhand accounts of dynamic systems, it is difficult to ascertain the authors' understanding and the adherence to Newell's model of DST. The idea of dynamic systems stems from the early work of Bernstein [22] in describing the nonlinear dynamics of movement. Kugler et al. [77] furthered this work to formulate the modern trajectory of understanding movement coordination. From this initial study, different lines of research have taken a variety of viewpoints as to how this relationship exists, Newell's model [14] being one of them. In DST, according to Newell [14], there is an equal interdependent relationship between each of the constraints to spontaneously organize in the form of a behavior; not all lines take this exact view point, although they are interested in the dynamics of coordination. This review was interested in how researchers have used the theory itself, as proposed by Newell [14], to inform practice; therefore, this article, among others, was excluded. A larger review is necessary to understand how constraints and dynamics outside of DST are being utilized in the bulk of research.

\section{Conclusion}

Despite a limited number of empirical studies utilizing Newell's model as a framework for intervention, a number of articles have referenced DST as a potentially promising theory to guide intervention [71, 72]. This review provides an aggregated view of the research regarding DST and motor development; until this point, most suggestions have not included the entire body of research on this topic. In this review, only one intervention article was identified; the rest were interested in validating the idea of how constraints influence motor behavior. The intervention article was published very recently [61] and suggests a possible increase in the interest of motor development and the potential effect of DST. As demonstrated earlier, motor development research appears to occur in cycles, with each generation seemingly figuring out how everything works, to then later be addressed and improved upon. Newell and Jordan [15] stated that more research is needed to understand DST's place in the development of motor skills and potential use for intervention through the manipulation of constraints [72]. Despite the lack of research formally utilizing DST according to Newell [14], many articles provide suggestions as to how constraints can be used to modify performance [72] in a variety of populations, including patients recovering from strokes [78] to children with autism [79] and on skills ranging from swimming [80] to language development [81].

DST provides a practical view of how behavior occurs without attributing the occurrence to any one subsystem but an active and fluid interaction between multiple elements. This interaction currently best explains the nuances of behavior caused by individual variability. As far as motor movement is concerned, it is evident that this behavior occurs through the influences of more than just a neural, predesigned 'program'. Individual motor movement and development can be and is affected by the constraints present at the time. This interaction of constraints can be of potential benefit to researchers and practitioners looking to improve motor skills. As shown 
in several studies [20,52, 57, 59], a manipulation of just one constraint can cause a spontaneous reorganization of the other constraints to produce a new behavior. If done purposefully, the manipulation of constraints can provide a powerful intervention to influence motor movement for the better [61].

This type of intervention can be extremely beneficial for children with ASD, or any disability for that matter, as the modified constraint manipulates the behavior without a necessary influence from the individual. As children with ASD often display motor impairments, it is imperative that a motor intervention be developed to counteract this delayed development, as it could have repercussions for individuals with ASD in the future [82]. As the hallmark of ASD is a deficit in social communication [35], typical instruction and modeling have little effect on the motor output of the individual. DST provides a framework for influencing behavior beyond verbal instruction or physical interaction. By manipulating how the individual performs a task or the environment it is performed in, practitioners and researchers can have an influence on the motor output and, thus, work to build an intervention of modifications that influence a more efficient and mature movement pattern.

\section{References}

1. Kamm K, Thelen E, Jensen JL. Dynamical systems approach motor development. Phys Ther 1990;70:763-75.

2. Gabbard CP. Lifelong motor development, 5th ed. New York: Pearson, 2008.

3. Payne VG, Isaacs LD. Human motor development, 6th ed. New York: McGraw Hill, 2005.

4. Darwin CR. A biographical sketch of an infant. Mind 1877;2:285-94.

5. Shinn MW. The biography of a baby. Boston: Houghton Mifflin, 1900.

6. Roberton MA. Motor development: recognizing our roots, charting our future. Quest 1989;41:213-23.

7. Thelen, E. Motor development. Am Psychol 1995;50:79-95.

8. Gabbard C, Krebs R. Studying environmental influence on motor development in children. Phys Ed 2012;69:136-49.

9. Clark JE, Whitall J. What is motor development? The lessons of history. Quest 1989;41:183-202.

10. Stelmach GE. Information processing in motor control and learning. New York: Academic Press, Inc., 1978.

11. Thelen E, Ulrich BD. Hidden skills. Monogr Soc Res Child Dev 1999:56:6-15.

12. Lewis MD. The promise of dynamic systems approach for an integrated account of human development. Child Dev 2000;71:36-43.

13. Clark JE, Phillips SJ. A longitudinal study of intralimb coordination in the first year of independent walking. Child Dev 1993;64:1143-57.
14. Newell KM. Constraints of the development of coordination. In: Wade MG, Whiting H, editors. Motor development in children: aspects of coordination and control. Dordrecht, The Netherlands: Martinus Nijhoff, 1986:341-60.

15. Newell KM, Jordan K. Task constraints and movement organization: a common language. In: Davis WE, Broadhead GD, editors. Ecological task analysis and movement. Human Kinetics, 2007:5-13.

16. Haywood KM, Getchell N. Life span motor development, 4th ed. Champaign, IL: Human Kinetics, 2004.

17. Langley DJ. The influence of functional constraints on sport-skill learning in a senior adult. J Aging Phys Act 2001:9;269-84.

18. Hutzler Y. A systematic ecological model for adapting physical activities: theoretical foundations and practical examples. Adapt Phys Activ Q 2007:24;287-304.

19. Gagen LM, Getchell N. Using “constraints" to design developmentally appropriate movement activities for early childhood education. Early Child Educ J 2006;34:227-32.

20. Renshaw I, Chow JY, Davids K, Hammond J. A constraints-led perspective to understanding skill acquisition and game play: a basis for integration of motor learning theory and physical education praxis? Phys Educ Sport Peda 2010;15:117-37.

21. Hadders-Algra M. Variation and variability. Phys Ther 2010;90:1823-37.

22. Berstein NA. The co-ordination and regulation of movements. Oxford, New York: Pergamon Press, 1967.

23. Turvey MT. Coordination. Am Psychol 1990;45:938-53.

24. Davids K, Glazier P, Araújo D, Bartlett R. Movement systems as dynamic systems: the functional role of variability and its implications for sport medicine. Sport Med 2003;33:245-60.

25. Šerbetar I. The role of dynamic systems in motor development research. Ann Kinesiol 2014;5:113-21.

26. Ulrich BD. Opportunities for early intervention, based on theory, basic neuroscience and clinical science. Phys Ther 2010;90:1868-80.

27. Wicke J, Jensen R. A pilot study of a dynamical systems approach to examining changes in static balance of adolescents. Percept Mot Skills 2002;95:267-78.

28. Gagen L, Getchell N. Combining theory and practice in the gymnasium "constraints" within an ecological perspective. J Phys Educ Recr Dance 2004;75:25-30.

29. Liu T, Hamilton M, Davis L, ElGarhy S. Gross motor performance by children with autism spectrum disorder and typically developing children on TGMD-2. J Child Adol Behav 2014;2:1-4.

30. Pan CY, Tsai CL, Chu CH. Fundamental movement skills in children diagnosed with autism spectrum disorders and attention deficit hyperactivity disorder. J Autism Dev Disord 2009;39:1694-705.

31. Staples KL, Reid G. Fundamental movement skills and autism spectrum disorders. J Autism Dev Disord 2010;40:209-17.

32. Liu T. Motor milestone development in young children with autism spectrum disorders: an exploratory study. Educ Psychol Pract 2012;28:315-26.

33. Lloyd M, MacDonald M, Lord C. Motor skills of toddlers with autism spectrum disorders. Autism 2013;17:133-46.

34. Ozonoff S, Young GS, Goldring S, Greiss-Hess L, Herrera AM, Steele J, et al. Gross motor development, movement abnormalities, and early identification of autism. J Autism Dev Disord 2008;38:644-56. 
35. Staples KL, MacDonald M, Zimmer C. Assessment of motor behavior among children and adolescents with autism spectrum disorder. Int Rev Res Dev Disabil 2012;42:179-214.

36. Fournier KA, Hass CJ, Naik SK, Lodha N, Cauraugh JH. Motor coordination in autism spectrum disorders. J Autism Dev Disord 2010;40:1227-40.

37. Dewey D, Cantell M, Crawford SG. Motor and gestural performance in children with autism spectrum disorders, developmental coordination disorder, and/or attention deficit hyperactivity disorder. J Int Neuropsychol Soc 2007;13:246-56.

38. Wong C, Odom SL, Hume K, Cox AW, Fettig A, Kucharczyk S, et al. Evidence-based practices for children, youth, and young adults with autism spectrum disorder. Chapel Hill: The University of North Carolina, Frank Porter Graham Child Development Institute, Autism Evidence-Based Practice Review Group, 2013.

39. American Psychological Association (APA). Diagnostic and statistical manual of mental disorders, 5th ed. Washington, DC: Author, 2013.

40. Colombo-Dougovito AM. Successful evidence-based practices for autism spectrum disorder and their use for the development of motor skills in physical education. Palaestra 2015;29:34-41.

41. Healy S. Adapting equipment. Palaestra 2014;27:37-42.

42. Breslin CM, Rudisill ME. The effect of visual supports on performance of the TGMD-2 for children with autism spectrum disorder. Adapt Phys Activ Q 2011;28:342-53.

43. Breslin CM, Rudisill ME. Relationships among assessment time, time on task, and motor skill performance in children with autism spectrum disorder. Adapt Phys Activ Q 2013;30:338-50.

44. Stodden DF, Gao Z, Goodway JD, Langendorfer SJ. Dynamic relationships between motor skill competence and health-related fitness in youth. Pediatr Exerc Sci 2014;26:231-41.

45. MacDonald M, Jaszewski C, Esposito P, Ulrich D. The effect of learning to ride a two-wheel bicycle on the social development of children with autism spectrum disorder. Palaestra 2011;25:37-42.

46. Bedford R, Pickles A, Lord C. Early gross motor skills predict the subsequent language development of language in children with autism spectrum disorder. Autism Res 2015;13:15-24.

47. Abney DH, Warlaumont AS, Haussman A, Ross JM, Wallot S. Using nonlinear methods to quantify changes in infant limb movements and vocalizations. Front Psychol 2014;5:1-16.

48. Astill S. Can children with developmental coordination disorder adapt to task constraints when catching two-handed? Disabil Rehabil 2007;29:57-67.

49. Bennett S, Button C, Kingsbury D, Davids K. Manipulating visual informational constraints during practice enhances the acquisition of catching skill in children. Res Q Exerc Sport 1999;70:220-32.

50. Clemente F, Couceiro M, Martins F, Dias G, Mendes R. Influence of task constraints on attacker trajectories during $1 \mathrm{~V} 1 \mathrm{sub}$ phases in soccer practice. Sportlogia 2012;8:13-20.

51. Farrow $D$, Reid $M$. The effect of equipment scaling on the skill acquisition of beginning tennis players. J Sports Sci 2010;28:723-32.

52. Langendorfer S. Motor-task goal as a constraint on developmental status. In: Clark JE, Humphery JH, editors. Advances in motor development research, 3rd ed. New York, NY: AMS Press, Inc., 1990:16-28.

53. Langendorfer SJ, Roberton MA. Individual pathways in the development of forceful throwing. Res Q Exerc Sport 2002:73:245-56.
54. Liu Y-T, Mayer-Kress G, Newell KM. Qualitative and quantitative change in the dynamics of motor learning. J Exp Psychol Hum Percept Perform 2006;32:380-93.

55. Maida SO, Mccune L. A dynamic systems approach to the development of crawling by blind and sighted infants. Review 1996;28:119-36.

56. Ohgi S, Loo KK, Morita S, Mizuike C. A dynamical systems analysis of spontaneous movements in newborn infants. J Mot Behav 2007;39:203-14.

57. Stergiou N, Jensen JL, Bates BT, Scholten SD, Tzetzis G. A dynamical systems investigation of lower extremity coordination during running over obstacles. Clin Biomech 2001;16:213-21.

58. Sweeting T, Rink JE. Effects of direct instruction and environmentally designed instruction on the process and product characteristics of a fundamental skill. J Teach Phys Educ 1999;18:216-33.

59. Ulrich BD, Ulrich DA, Angulo-Kinzler RM. The impact of context manipulations on movement patterns during a transition period. Hum Mov Sci 1998;46:327-46.

60. Ulrich BD, Ulrich DA, Collier DH, Cole EL. Developmental shifts in the ability of infants with down syndrome to produce treadmill steps. Phys Ther 1995;75:14-23.

61. Vernadakis N, Papastergiou M, Zetou E, Antoniou P. The impact of an exergame-based intervention on children's fundamental motor skills. Comput Educ 2015;83:90-102.

62. Volman M, Wijnroks A, Vermeer A. Effect of task context on reaching performance in children with spastic hemiparesis. Clin Rehabil 2002;16:684-92.

63. Wu C-Y, Lin K-C, Lin K-H, Chang C-W, Chen C-L. Effects of task constraints on reaching kinematics by healthy adults. Percep Mot Skills 2005;100:983-94.

64. Balan CM, Davis WE. Ecological task analysis. J Phys Educ Recr Dance 1993;64:54-61.

65. Burton AW, Davis WE. Ecological task analysis. Hum Movement Series 1996:15;285-314.

66. Herkowitz J. Developmental task analysis: the design of movement experiences and evaluation of motor development status. In: Ridenour M, editor. Motor development issues and applications. Princeton, New York: Princeton Book Co, 1978:139-64.

67. Gesell A. Maturation and infant behavior pattern. Psychol Rev 1929;36:307-19.

68. McGraw MB. The neuromuscular maturation of the human infant. New York: Columbia University Press, 1943.

69. Clark JE. On becoming skillful: patterns and constraints. Res Q Exerc Sport 1995;66:173-83.

70. Clark JE, Metcalfe SS. The mountain of motor development: a metaphor. In: Clark JE, Humphrey JH, editors. Motor development: research and reviews, 2nd ed. Reston, VA: National Association of Sport and Physical Education, 2002:163-90.

71. Brymer E, Renshaw I. An introduction to the constraints-led approach to learning in outdoor education. Australian Journal of Outdoor Education 2010;14:33-41.

72. Coker CA. Intervention strategies for performance enhancement. Phys Health Educ 2014;79:14-7.

73. Clark JE. Development of voluntary motor skill. In: Meisami E, Timiras PS, editors. Handbook of human growth and development biology. Boca Raton, FL: CRC Press, 1999:237-50. 
74. Thelen E. The (re)discovery of motor development. Dev Psychol 1989;25:946-49.

75. Gibson JJ. The ecological approach to visual perception. Boston, MA: Houghton Mifflin, 1979.

76. Schmidt RA. A schema theory of discrete motor skill learning. Psychol Rev 1975;82:225-60.

77. Kugler PN, Kelso JA, Turvey MT. On the concept of coordinative structure as dissipative structures: I. Theoretical lines of convergence. In: Stelmach GE, editor. Tutorials in motor behavior. Amsterdam: North-Holland, 1980.

78. Sabari JS, Kane L, Flanagan SR, Stineberg A. Constraint-induced motor relearning after stroke: a naturalistic case report. Arch Phys Med Rehabil 2001;82:524-28.
79. Pope M, Liu T, Breslin CM, Getchell N. Using constraints to design developmentally appropriate movement activities for children with autism spectrum disorders. J Phys Educ Recr Dance 2012;83:35-41.

80. Seifert L, Komar J, Barbosa T, Toussaint H, Millet G, Davids K. Coordination pattern variability provides functional adaptations to constraints in swimming performance. Sport Med 2014;44:1333-45.

81. Spoelman M, Verspoor M. Dynamic patterns in development of accuracy and complexity. Appl Linguist 2010;31:532-53.

82. MacDonald M, Esposito P, Ulrich D. The physical activity patterns of children with autism. BMC Res Notes 2011;4:422. 